\section{MYS}

médecine/sciences $1992 ; 8: 900-1$

\title{
L'ADN, UN MÉDICAMENT POUR DEMAIN
}

\section{Axel Kahn Marc Peschanski}

\section{RÉFÉRENCES}

1. Kahn A, Briand P. Nouvelles orientations pour la thérapie génique. médecine/sciences $1990 ; 6: 144-9$

2. Kahn A, Briand P. Thérapie génique : espoirs et limitres. médecine/sciences 1991; 7 : 705-714.

3. Anderson WF. Human gene therapy. Science 1992 ; 256 : 808-813.

4. Miller AD. Human gene therapy comes of age. Nature $1992 ; 357$ : 455-60.

5. Van Beusechem V, Kukler A, Heidt P, et al. Long-term expression of human adenosine deaminase in rhesus monkeys transplanted with retrovirus-infected bone marrow cells monkeys transplanted with retrovirus-infected bne marrow cells. Proc Natl Acad Sci USA 1992 ; 89 : 7640-44.

6. Thompson L. At age 2, gene therapy cnters a growth phase. Science $1992 ; 258$ : 744-6.

7. Jiao $\mathrm{S}$, Williams $\mathrm{P}$, Berg $\mathrm{RK}$, et al. Direct gene transfer into nonhuman primate myofibers in vivo. Hum Gene Ther 1992b ; 3 : 21-33.

8. Li M, Hantzopoulos PA, Banerjec D, et al. Comparison of the expression of a mutant dihydrofolate reductase under control of different internal promoters in retroviral vectors. Hum Gene Ther 1992; 3 : 381-90.

9. Stratford PL, Levrero M, Chasse JF, et al. Evaluation of the transfer and expression in mice of an enzyme-encoding gene using a human adenovirus vector. Hum Gene Ther $1990 ; 1: 241-56$

\section{ADRESSES}

A. Kahn : directeur de l'inserm U. 129. ICGM, unité de génétique et de pathologie moléculaires, Inserm U. 129, 24, rue du Faubourg-Saint-Jacques, 75014 Paris, France.

M. Peschanski : directeur de recherche à l'Inserm. CJF 91,02, neuroplasticité et greffes intracérébrales, centre hospitalo-universitaire Henri Mondor, 51, avenue du Maréchal-de-

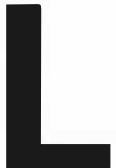

e concept de thérapie génique, c'est-à-dire l'utilisation directe de l'ADN à des fins thérapeutiques, continue de bénéficier d'une considérable popularité $[1,2]$. C'est que la reprogrammation génétique des cellules malades qu'elle se fixe pour objectif tend vers le magique qui a enluminé nos rêves d'enfant. Le thérapeute généticien capable de modifier le destin héréditaire d'une cellule génétiquement vouée à causer la souffrance est perçu comme ayant ce pouvoir qui permettait aux magiciens des contes d'antan de transformer la bête en prince charmant. De ce fait, la moindre introduction du moindre fragment d'ADN dans la moindre cellule humaine fait les grands titres de la presse, des revues scientifiques aux quotidiens d'inf ormation. On perd souvent de vue, ce faisant, qu'une grande partie des transferts in vivo d'ADN chez l'homme a été réalisé et est réalisé hors de tout objectif thérapeutique et, de ce fait, ne mérite en aucun cas l'appellation de thérapie génique. Ces essais ont en général pour but de marquer, par introduction d'un gène, des cellules dont on veut suivre le destin dans l'organisme, afin d'apprécier l'efficacité d'un traitement ou de comprendre les raisons de son échec. L'intérêt de ces premières expériences de transfert d'ADN chez l'homme est cependant considérable pour l'avenir des thérapies géniques, d'abord sur le plan psychologique puisqu'il permet d'accoutumer l'opinion à l'idée du transfert de gènes dans des cellules humaines et de bien marquer la différence entre ce type d'expérience et l'idée fantasmagorique que se font certains des " manipulations génétiques". Par ailleurs, mais cela n'était pas inattendu au vu des nombreux essais chez l'animal qui avaient précédé, il est apparu que, dans les protocoles utilisés, aucun effet néfaste de l'introduction de cellules génétiquement modifiées ne pouvait être observé $[3,4]$. Une autre information tirée du marquage génétique des TIL (tumor infiltrating lymphocytes), utilisés pour leur effet anti-cancéreux dans des mélanomes ou des cancers du rein, est que ces cellules ne semblent pas fonctionnellement affectées par la modification génétique et ont une durée de vie extrêmement longue. La seule vraie thérapie génique réalisée chez l'homme et dont on puisse, à ce jour, analyser les résultats, est le transfert du gène de l'adénosine désaminase dans les lymphocytes de petites malades présentant un syndrome d'immunodéficience combinée par carence en cette enzyme. Les résultats sont ici difficiles à analyser car les sujets traités ont continué de recevoir des préparations enzymatiques de PEG-ADA, seul traitement, avec la greffe de moëlle, antérieurement disponible pour traiter ces malades. Cependant, une nette amélioration clinique et biologique semble avoir été obtenue au prix d'injections renouvelées, d'abord tous les mois, puis tous les cinq mois [3]. L'innocuité de ce traitement a pu être confirmé, de même que la longue durée de vie de certaines des cellules injectées. En dehors de ce succès, au moins partiel, aucune des tentatives actuellement en cours de thérapie génique chez l'homme n'en est à un stade suffisant pour que l'on ait la moindre idée de son efficacité. Ces essais concernent l'hypercholestérolémie familiale, traitée par réimplantation d'hépatocytes infectés ex vivo par un vecteur rétroviral véhiculant le gène du récepteur des LDL ; l'injection de fibroblastes préalablement infectés par un rétrovirus recombinant portant l'ADNc du facteur IX ; le transfert in vivo par des liposomes d'ADN codant pour une molécule du complexe majeur d'histocompatibilité ; et, enfin, une prolongation des essais de Rosenberg et al. dans laquelle le gène marqueur introduit dans les TIL est remplacé par (ou associé à) des gènes de cytokines, TNF ou IL2 ( $\mathrm{m} / \mathrm{s} n^{\circ} 1$, vol. 8, p. 80) [3, 4]. Cependant, un nombre bien plus grand de protocoles d'essais thérapeutiques a été déposé devant les autorités compétentes, principalement aux États-Unis mais aussi dans les autres pays, notamment la France; plusieurs essais devraient débuter ainsi très prochainement. On ne peut, pour prévoir leurs chances de succès, que se reposer sur l'expérimentation animale. Une des grandes leçons récentes tirées de celle-ci est que certaines informations obtenues sur l'utilisation de modèles particuliers 
sont probablement difficilement transposables à une autre espèce, notamment à l'homme. C'est ainsi que la greffe de moelle autologue infectée ex vivo par un rétrovirus recombinant donne des résultats bien supérieurs chez la souris que chez les gros animaux, chien, chat et singe [4]. Dans cette dernière espèce, un article récent rapporte que seulement 1 à $2 \%$ des cellules sanguines expriment un transgène introduit de cette façon, ces résultats tendant à se dégrader au fil du temps [5]. Cependant, des résultats nettement supérieurs ont été obtenus en transférant un gène marqueur dans les cellules médullaires de cancéreux pré-traités par chimiothérapie et soumis à une auto-greffe de moelle [6]. L'injection intra-musculaire directe d'ADN plasmidique recombiné semble également aboutir à des niveaux d'expression nettement supérieurs chez les rongeurs que chez les primates [7]. Les constructions génétiques, c'est-àdire les promoteurs et les séquences régulatrices assurant les meilleurs niveaux d'expression, seraient également différentes selon l'espèce dans laquelle le transgène doit être transféré [8]. C'est arguant de cette difficulté à reproduire sur d'autres espèces, et donc probablement sur l'homme, les résultats acquis sur un petit animal de laboratoire, que de très nombreuses équipes ont tendance aujourd'hui à passer directement à l'expérimentation humaine. Reste cependant à prouver qu'un résultat peut s'avérer positif chez les primates et chez l'homme alors qu'il a été complétement négatif sur les rongeurs de laboratoire. C'est chez ces derniers qu'ont été obtenus les seuls résultats complètement probants, à ce jour, de l'efficacité de la thérapie génique somatique : correction du phénotype lié à un déficit en ornithine transcarbamylase murine à l'aide d'un vecteur adénoviral [9] ; régression de la surcharge cellulaire, splénique et hépatique, chez les souris déficientes en $\beta$-glucuronidase par infection de cellules médullaires ou de fibroblastes formant des organoïdes [1] par des rétrovirus recombinés (O. Danos et J.M. Heard, Institut Pasteur, Paris, communication personnelle); diminution de la bilirubine et apparition de ses dérivés conjugués chez le rat Gunn par transduction rétrovirale du gène de la bilirubine glucuronyltransférase dans les hépatocytes (O. Danos et J.M. Heard, communication personnelle) ; amélioration très partielle de l'hypercholestérolémie chez le lapin Watanabe par transduction rétro$\mathrm{m} / \mathrm{s} n^{\circ} 9 \mathrm{vol} .8$, novembre 92 virale d'hépatocytes réinjectés dans le système porte $\left(\mathrm{m} / \mathrm{s}, n^{\circ} 5\right.$, vol. $8, p$. 499) et, surtout, destruction in situ des cellules d'un glioblastome par transfert dans le cerveau de cellules produisant un rétrovirus recombiné pour le gène de la thymidine kinase du virus $\mathrm{HSV}(\mathrm{m} / \mathrm{s}$, $n^{\circ} 5$, vol. 8, p. 798). C'est sur la base de ces succès que des protocoles d'essais chez l'homme ont été déposés (voire sont en cours, pour l'hypercholestérolémie). Une autorisation a été donnée à l'équipe de Michael Blaese pour le traitement de 20 glioblastomes inopérables chez l'homme. Le principe du traitement des tumeurs cérébrales par des rétrovirus véhiculant le gène HSV-tk est l'infection exclusive des cellules malignes, c'est à dire des seules cellules prolifératives dans le contexte du système nerveux central. C'est dire que cette technique n'est pas directement applicable au transfert de gènes dans les cellules normales du cerveau, notamment dans les neurones qui ne se divisent plus du tout après la naissance. Or les maladies neurologiques constituent un champ d'applications potentiel considérable des traitements par transfert de gènes. C'est que, dans ce domaine, les moyens thérapeutiques existants sont le plus souvent encore très rudimentaires, justifiant les essais thérapeutiques en cours de transfert de cellules, en particulier de neurones embryonnaires $(\mathrm{m} / \mathrm{s}$ $n^{\circ} 6$, vol. 8, p. 601).

Le transfert de gènes dans les cellules post-mitotiques du cerveau est aujourd'hui possible grâce aux vecteurs HSV-1 (voir l'article d'A. Epstein, p. 902 de ce numéro) et adénoviraux (résultats non publiés de nos laboratoires), alors que d'autres vecteurs sont à l'étude. Lorsque seront réglés les problèmes de cytopathogénicité résiduelle et de stabilité d'expression, on pourra sérieusement s'attacher à définir les conditions de leur utilisation thérapeutique. Il est peu probable que l'on envisage dans un premier temps une véritable reprogrammation neuronale visant à corriger une expression génétique pathologique comme dans la chorée de Huntington ou l'ataxie de Friedreich. Outre la faiblesse des connaissances génétiques, on se heurte ici aux problèmes, paradoxalement complémentaires, de la nécessaire dispersion du transgène à une large population de neurones - ce qui n'est pas réalisé par les vecteurs actuels - et du ciblage obligatoire vers des neurones précis, ce qui nécessitera l'utilisation, aujourd'hui difficile, de promoteurs spécifiques. Bien plus probable- ment, les premiers transferts d'ADN dans le cerveau ne viseront qu'à faire produire par des cellules quelconques une protéine thérapeutique sous le contrôle d'un promoteur ubiquitaire, fort. On peut donner trois objectifs différents à cette thérapie génique : (1) la compensation d'un déficit biochimique par introduction d'un gène codant pour une enzyme appartenant à une chaîne métabolique - par exemple dans des maladies lysosomiales [10] — ou à une chaîne de synthèse d'un neurotransmetteur - tyrosine hydroxylase dans la maladie de Parkinson, choline acétyltransférase dans la maladie d'Alzheimer ; (2) la modulation d'une activité neuronale qui a perdu ou dépassé les contrôles naturels (exemple de l'épilepsie ou de la douleur chronique) ; (3) la protection neuronale contre les agressions. Ces dernières années ont vu, en effet, l'émergence d'une nouvelle classe de substances, les facteurs " neurotrophiques" dont certains semblent capables de protéger des populations plus ou moins spécifiques de neurones contre les conséquences létales d'atteintes traumatiques (exemple du NGF pour les neurones chlolinergiques septaux) [11] ou d'origine génétique [12]. L'analyse du génome a pris, depuis peu, une accélération fantastique qui permet presque de dater à quelques années son aboutissement. La thérapie génique apparaît comme une des retombées majeures de ce gigantesque effort de génétique moléculaire, et comme un retour dialectique des plus logiques vers la physiologie et la physiopathologie. Pour paraphraser les bons auteurs, on ne peut plus aujourd'hui se contenter de comprendre le monde, il faut chercher à le transformer!

\section{RÉFÉRENCES}

10. Dreyfus JC, Akli S, Pcenaru L. Maladies de Tay-Sachs et de Sandhoff : les déficits en $\beta$-hexosaminidases, modèles de maladies des lysosomes. médecine/sciences $1992 ; 8$ : 797-804

11. Brachet P. Le facteur de croissance nerveuse NGF : rôle dans la plasticité et la maintenance fonctionnelle de la cellule neuronale. médecine/sciences $1990 ; 6: 854-62$. 12. Sendtner M, Schmalbruch $\mathrm{H}$, Stockli $\mathrm{KA}$, et al. Ciliary neurotrophic factor prevents degenerations of motor neurons in mouse mutant progressive motor neuronopathy. Nature $1992 ; 358$ : 502-4.

TIRÉS A PART

A. Kahn 